\title{
Chemische Hydrogelsensoren auf Basis des Bimorpheffekts mit kurzer Ansprechzeit
}

\author{
Stefan Schreiber $^{1}$, Nadja Steinke ${ }^{2}$ und Gerald Gerlach ${ }^{1}$ \\ 1 Institut für Festkörperelektronik, Technische Universität Dresden, Dresden, Deutschland \\ ${ }^{2}$ Fraunhofer Institut für Keramische Technologien und Systeme (IKTS), Dresden, Deutschland \\ Kontakt: stefan.schreiber@tu-dresden.de
}

\section{Einleitung}

Elastische Polymernetzwerke (Hydrogele) weckten erstmals 1949 das Interesse zur Anwendung als Aktor [1]. Wegen ihres hydrophilen Charakters quellen Hydrogele aus dem Trockenem, wenn sie mit Wasser in Kontakt kommen. Responsive Hydrogele reagieren aufgrund funktioneller Gruppen zusätzlich auf externe Stimuli, wie u. a. Ionenkonzentration oder Temperatur.

Detektiert man die von der Quellung hervorgerufene Volumenänderung mit einem geeignetem Sensor, z. B. einem piezoresistiven Drucksensor, wird der entsprechende Stimulus messbar [2]. Durch Variation der Polymerzusammensetzung lassen sich anstatt der Ionenkonzentration auch Konzentrationen anderer gelöster Stoffe, wie zum Beispiel Urea, Ethanol oder Ammoniak [3]-[5], messen.

Für eine funktionelle Anwendung sind gegenwärtig die Quell- und Entquellzeiten der Hydrogele und somit die Messzeiten der Sensoren meist noch zu langsam. Eine Verkürzung der Antwortzeiten lässt sich durch verschiedene Maßnahmen erreichen. Zum Beispiel erhöht eine poröse Hydrogelstruktur die Quellgeschwindigkeit [6] und durch Anwendung der Kraftkompensationsmethode kann das Eindringen der Messlösung weitestgehend verhindert und so die Kinetik erheblich beschleunigt werden. Damit erreicht man eine Ansprechzeit im Bereich weniger Minuten [7].

In dieser Veröffentlichung soll vorgestellt werden, wie die Ansprechzeit des Hydrogels noch weiter bis auf wenige Sekunden reduziert werden kann. Besonders starken Einfluss auf die Quellkinetik haben die Diffusionskonstante und das Volumen des Hydrogels. Ein reduziertes Hydrogelvolumen, insbesonders eine deutlich verringerte Dicke, verkürzt die Diffusionsstrecke des Analyts und somit die Zeit, bis das Hydrogel vollständig gequollen ist.
Die Volumenänderung dünner Hydrogele, bzw. der entsprechende Quelldruck, lässt sich mittels piezoresistiver Sensoren und des Bimorph-Effekts messen [8]. Hierbei wird das Hydrogel sehr dünn auf die Biegeplatte eines piezoresistiven Drucksensor aufgebracht und lokal strukturiert. Der Bimorph-Effekt bewirkt eine Auslenkung der Biegeplatte und damit ein elektrisches Messsignal. Erste Ergebnisse zeigen, dass sich auf diese Weise die Einschwingzeiten auf wenige Sekunden verkürzen lassen. Durch die Kombination mit der Kompensationsmethode lassen sich die Messzeiten noch weiter verkürzen.

\section{Aufbau und Methoden}

Abbildung 1 zeigt schematisch den Aufbau des verwendeten Sensors. Kernelement ist ein piezoresistiver Drucksensor (C41, Firma TDK) mit einem Messbereich von 0-25 mbar. Außerdem befindet sich direkt auf dem Chip eine Diode zur Messung der Temperatur. Auf der Innenseite der Membran, in der Kavität, ist das Hydrogel strukturiert. Der Sensorchip selbst wird auf einem TO-8 Sockel aufgeklebt, sodass sich zwischen Drucksensor und Sockel eine fluidische Kammer bildet. Diese lässt sich über die Fluidkanäle mit dem Analyt befüllen.

Das Hydrogel ist bisensitiv, sodass es sowohl infolge einer sich hier beispielhaft änderenden Salzkonzentration, als auch durch eine Temperaturänderung quellen bzw. entquellen kann. Ziel ist nun, durch eine angepasste Temperaturregelung die durch Salzkonzentration verursachten Quellung so zu kompensieren, dass das Volumen

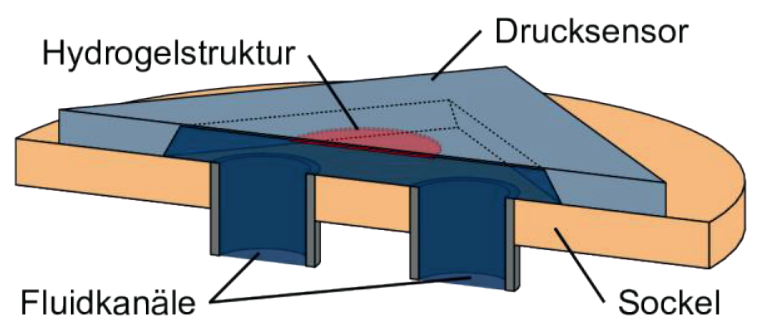

Abb. 1: Schematischer Aufbau des Hydrogelsensors 
konstant bleibt, um möglichst alle Driftprozesse zu vermeiden.

\section{Hydrogelzusammensetzung}

Das bisensitive Hydrogel besteht aus den Monomeren $\mathrm{N}$-Isopropylacrylamid (NIPAAm) und 2-Acrylamido-2-methylpropansulfonsäure (AMPS). Polymerisiertes und vernetztes PNIPAAm reagiert auf Temperaturänderungen. Steigt die Temperatur des Hydrogels, so entquillt PNIPAAm und vice versa. PAMPS reagiert auf den Anstieg der Salzkonzentration mit Quellen. Für die Vernetzung der Polymerstränge wird N,N'-Methylenbisacrylamide (BIS) verwendet. Die Polymerisation wird mit dem photosensitiven Initiator LithiumPhenyl-2,4,6-trimethylbenzoylphosphinat (LAP) und einer Bestrahlung mit UV-Licht gestartet. Um dem hohen Säuregehalt der Pregel-Lösung auszugleichen, wird gelöstes Natriumhydroxid $(\mathrm{NaOH})$ hinzugegeben. Die prozentuale Zusammensetzung der Stoffe sind in Tabelle 1 aufgeführt.

Tab. 1: Hydrogelzusammensetzung

\begin{tabular}{lr}
\hline Stoff & Stoffmengenverhältnis $\mathbf{n} \%$ \\
\hline NIPAAm & 97,00 \\
AMPS & 5,73 \\
BIS & 4,00 \\
NaOH-Lsg. & 6,24 \\
LAP & 0,50 \\
\hline
\end{tabular}

\section{Sensorvorbereitung}

Damit die Hydrogelstruktur mit der Membran eine Verbindung eingeht, bedarf es eines Haftvermittlers in Form einer selbstorganisierenden Monomerschicht. Verwendet wird in Isopropanol gelöstes 3-Aminopropyltriethoxysilane (APTES). Die Silangruppe bindet sich an die Sauerstoffatome des Siliziumdioxids und bildet somit eine Lage abstehender Monomerketten, an welche sich das Hydrogel bei der Polymerisierung anbindet.

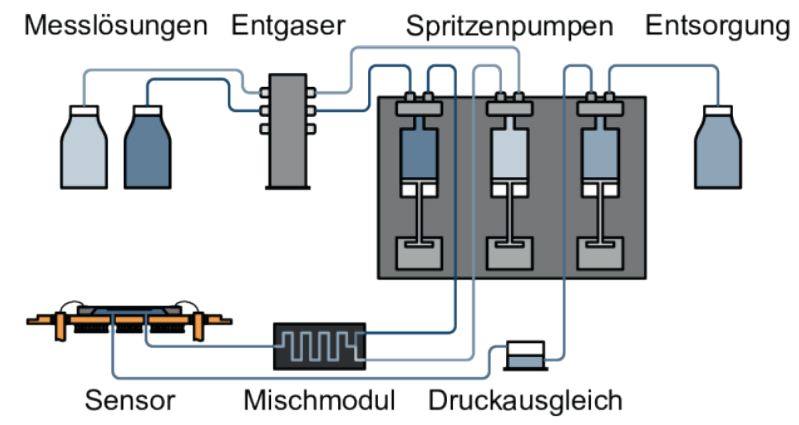

Abb. 2: Fluidischer Aufbau des Messplatzes

\section{Strukturierung}

Die Struktur des Hydrogels beeinflusst sowohl die Richtung als auch Stärke der Auslenkung infolge des Bimorpheffekts. Werden zwei Platten mit unterschiedlichen Längenausdehnungskoeffizienten verbunden, so bewirkt die Längenänderung einen Ausschlag des Verbunds in lateraler Richtung. Im Fall des Hydrogelsensors bilden Hydrogelstruktur und Siliziumplatte den Verbund. Die angestrebte Hydrogelstruktur ist eine Zylinderscheibe mit einer Höhe von $20 \mu \mathrm{m}$ und einem Durchmesser von $1,65 \mathrm{~mm}$. Es hat sich gezeigt, dass ein Verhältnis der Durchmesser von Hydrogel zur Biegeplatte von etwa 1:2 optimal für eine möglichst große Auslenkung ist [9].

Für die Strukturierung des Hydrogels wird ein Mikrokontaktprinter $\mu \mathrm{CP} \quad 4.1$ (GeSiM mbH, Großerkmannsdorf) verwendet.

Im ersten Schritt wird ein Poly(dimethysiloxan) (PDMS)-Stempel aus einem Master, der die Form der Hydrogelstruktur trägt, abgeformt. Die Kavität des Stempels wird mit der Hydrogel-Lösung befüllt und anschließend mithilfe des $\mu \mathrm{CPs}$ mit einem Druck von $20 \mathrm{kPa}$ für 1 min auf die Sensormembran gedrückt. Unter UV-Licht (Wellenlängenbereich $320 \mathrm{~nm}$ bis $500 \mathrm{~nm}$, OmniCure S1500, Excelitas Technologies $\mathrm{GmbH}$, Wiesbaden) wird die Struktur für 1 min polymerisiert.

Messplatz (fluidisch)

Zur Charakterisierung des Sensors wird der in Abbildung 2 dargestellte Messplatz verwendet. Betrieben wird dieser mit computergesteuerten automatischen Spritzenpumpen der Firma CETONI $\mathrm{GmbH}$. Als Messlösung werden eine Salzlösung $\left(1 \mathrm{~mol} / \mathrm{l} \mathrm{NaCl}\right.$ in $\mathrm{H}_{2} \mathrm{O}$ ) und destilliertes deionisiertes Wasser verwendet. Beide werden beim Aufziehen der Spritzen durch einen Entgaser DEGASi ${ }^{\circledR}$ Classic (dichrom $\mathrm{GmbH}$ ) geführt, um Gaseinschlüsse aus der Lösung zu entfernen. Die Spritzenpumpen dispensen anschließend die Messlösung durch ein Mikromischmodul in den Sensor. Da der Sensor auf kleinste Druckschwankungen reagiert, wird der Rücklauf vom Sensor in einen offenen Druckausgleichsbehälter geführt, von dem die Messlösung anschließend abgesaugt und in einen Entsorgungsbehälter gefördert wird.

\section{Messplatz (elektrisch)}

Der Sensor wird, wie in Abbildung 3 gezeigt, auf zwei Peltierelemente montiert. Diese werden über einen Peltier-Controller der Firma BelektroniG geregelt. Außerdem werden mit dem Controller 


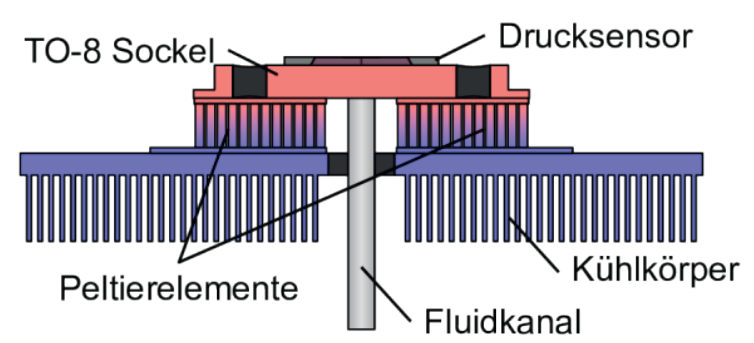

Abb. 3: Thermischer Aufbau des Messplatzes

Daten wie Sensorauslenkung und Temperatur von Sensorchip, Platine und Umgebung gemessen und aufgezeichnet.

\section{Messdatenaufzeichnung}

Spritzenpumpen und Peltierelemente werden mit der Software der jeweiligen Hersteller von einem Rechner aus gesteuert und überwacht. Beide Programme bieten eine Möglichkeit die Prozessdaten wie Pumpengeschwindigkeit, Ventilstellung und Sensordaten (Temperatur, Druck, ...) aufzuzeichnen und in Form einer CSVDatei zu speichern.

\section{Messdatenauswertung}

Zur Messdatenauswertung wird R [10], eine Software für statistische Berechnungen und Auswertungen, verwendet. Nach Einlesen der CSVDateien von Spritzenpumpe und Peltierregler werden die Datensätze anhand des Zeitstempels synchronisiert.

Zur Auswertung der Temperatursensitivität findet zunächst eine Unterteilung zwischen den unterschiedlichen Temperatursollwerten und der Sprungrichtung $\left(30^{\circ} \mathrm{C} \rightarrow 25^{\circ} \mathrm{C}\right.$ bzw. $\left.20^{\circ} \mathrm{C} \rightarrow 25^{\circ} \mathrm{C}\right)$ statt. Anschließend werden von jedem Sprung die Startzeiten subtrahiert, sodass die Sprungantworten bei $t=0$ beginnen und somit vergleichbar gemacht werden.

Für die $\mathrm{NaCl}$-Sensititvität werden die Pumpendaten benötigt. Die Unterteilung erfolgt hierbei entsprechend der von den Spritzen generierten Konzentration, also des Volumenstromverhältnisses der Pumpen. Über den Zeitstempel werden die Pumpendaten mit den Daten des Peltier-Controllers synchronisiert.

\section{Versuchsdurchführung}

Wie oben beschrieben, wird im Sensor ein bisensitives Hydrogel verwendet, welches auf Änderungen von Salzkonzentration und Temperatur mit einer Volumenänderung reagiert. Um gegenseitige Einflüsse zu minimieren, wird die jeweils andere Sensitivität gezielt konstant gehalten. Im Falle der Salzsensitivität wird vom Peltierregler die Temperatur im Sensorchip konstant gehalten, während beim Messen der Temperatursensitivität ein Analyt mit konstanter Konzentration durch den Sensor geführt wird.

\section{Temperatursensitivität}

Zur Charakterisierung der Temperatursensitivität wird der Sensor während der Messung mit $\mathrm{H}_{2} \mathrm{O}$ und einem konstanten Volumenstrom von $\dot{V}=0,1 \mathrm{ml} / \mathrm{min}$ gespült. Dies ist wichtig, da durch den Volumenstrom ein konstanter Wärmeabtransport entsteht, der in der Charakterisierung zu berücksichten ist. Anschließend wird durch die Software die Solltemperatur in dem Sensor eingestellt und die Antwort des Quellverhaltens aufgezeichnet.

\section{$\mathrm{NaCl}$-Sensitivität}

Für die Salzsensitivität wird der Sensor vom Regler der Peltierelemente auf einer konstanten Temperatur von $23^{\circ} \mathrm{C}$ gehalten, um den störenden Temperatureinfluss des Fluids, welches bei Raumtemperatur gelagert wird, zu minimieren. Der Messprozess beginnt mit dem Spülen des Sensors für 10 min mit $\mathrm{H}_{2} \mathrm{O}$. Dadurch sollen eventuelle Rückstände der letzten Messung und mögliche Lufteinschlüsse vom Sensorwechseln entfernet werden. Anschließend wird die $\mathrm{NaCl}$-Konzentration zwischen $0 \mathrm{~mol} / / \mathrm{l}$ und $1 \mathrm{~mol} / \mathrm{l}$ gewechselt. Nach der Messung wird erneut mit $\mathrm{H}_{2} \mathrm{O}$ gespült, damit sich die Salzkristalle nicht ablagern.

\section{Ergebnisse}

\section{Temperatursensitivität}

Für die Aufnahme der Temperatursensitivität wurde die Sensortemperatur minütlich zwischen $20^{\circ} \mathrm{C}$ und $30^{\circ} \mathrm{C}$ gewechselt. Abbildung 4 zeigt die ersten $20 \mathrm{~min}$ des Versuchs, welcher insgesamt 100 min lief und jeden Temperatursprung 50 mal wiederholte. Der dunkelblaue Graph zeigt die Temperaturantwort direkt vom Sensorchip und die rote Linie die vom Quellen des Hydrogels

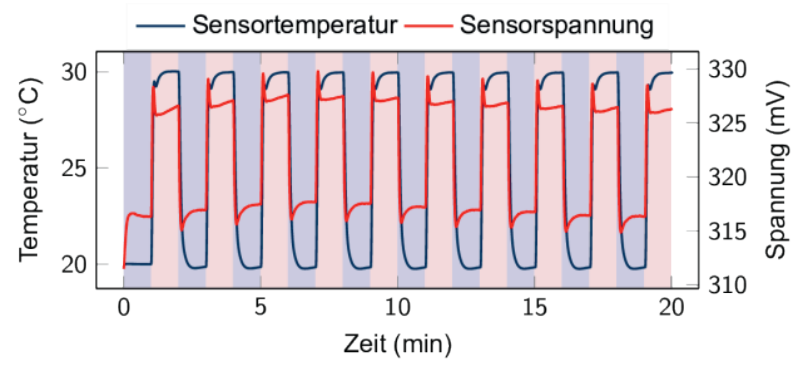

Abb. 4: Sensorsignal der ersten 20 min des Messdurchgangs. Die Solltemperatur wurde jede Minute zwischen $20^{\circ} \mathrm{C}$ und $30^{\circ} \mathrm{C}$ gewechselt 

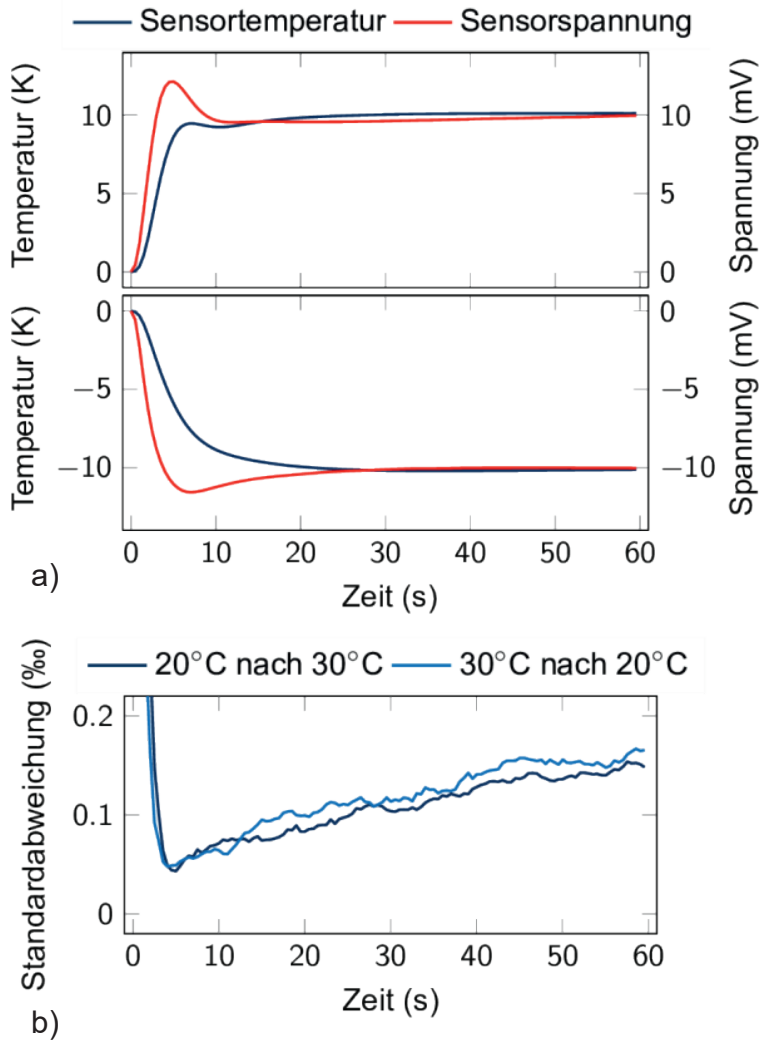

Abb. 5: a) Mittelwerte der Sprungantworten bei Erhöhung und Verringerung der Temperatur gemäß Abbildung 4 und b) zugerhörige Standardabweichungen

verursachte Änderung der Sensorspannung. Es lässt sich ein leichtes Driftverhalten der Grundlinie erkennen.

Fasst man die Temperatursprünge in einer Kurve zusammen und subtrahiert die Startwerte vom Sprung, kann man erkennen, dass das Sprungverhalten sehr reproduzierbar ist (Abbildung 5).

Betrachtet man die Standardabweichung der Sprünge in Abbildung 5b, lässt sich erkennen, dass mit zunehmender Dauer die Sprungantworten stärker streuen. Diese Streuung ist eine Erklärung für die bereits erwähnte Drift der Grundlinie. Die Ursachen dafür sind noch nicht gänzlich geklärt, es wird aber vermutet, dass die Umgebungs-

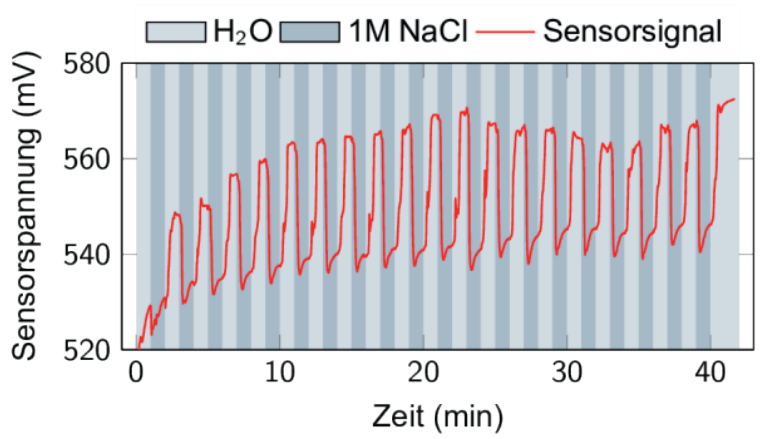

Abb. 6: Quellverhalten des Hydrogels beim zyklischen Wechsel der Konzentration zwichen $0 \mathrm{M}$ und $1 \mathrm{M} \mathrm{NaCl}$ temperatur und der Temperatur des Fluids trotz Regelung noch einen Einfluss auf das Messsignal haben.

Trotz der steigenden Streuung sind die Beträge der Standardabweichungen im Allgemeinen sehr gering. Dies deutet auf eine sehr hohe Reproduzierbarkeit der Sprungantworten des Hydrogels hin, deren Antwortzeiten mit weniger als $5 \mathrm{~s}$ erwartungsgemäß sehr gering ausfallen.

\section{Salzsensitivität}

Zur Messung der Salzsensitivität wird der Sensorchip konstant auf $23^{\circ} \mathrm{C}$ gehalten. Das Wechseln der Konzentration wird mit einem Programm für die Spritzenpumpen realisiert. Abbildung 6 zeigt das Quellverhalten des Hydrogels in Abhängigkeit von der Konzentrationsänderung, welche jeweils nach einer 1 min geändert wird. Es ist zu erkennen, dass das Hydrogel auf die Konzentrationsänderungen schnell reagiert, die Sprungantworten jedoch einer zeitlichen Drift unterliegen (Abbildung 6).

Zur Auswertung wurden die Sprungantworten nach Konzentrationswechsel zusammengefasst und deren Mittelwerte und Standardabweichung ermittelt (Abbildung 7).

\section{Diskussion}

Die Messergebnisse zeigen deutlich ein reproduzierbares Quellverhalten. Dies lässt darauf schließen, dass die störenden Einflüsse systematischer Natur sind und eliminiert werden können.

Relevante Einflüsse sind die Umgebungstemperatur des Sensors und die Temperatur des

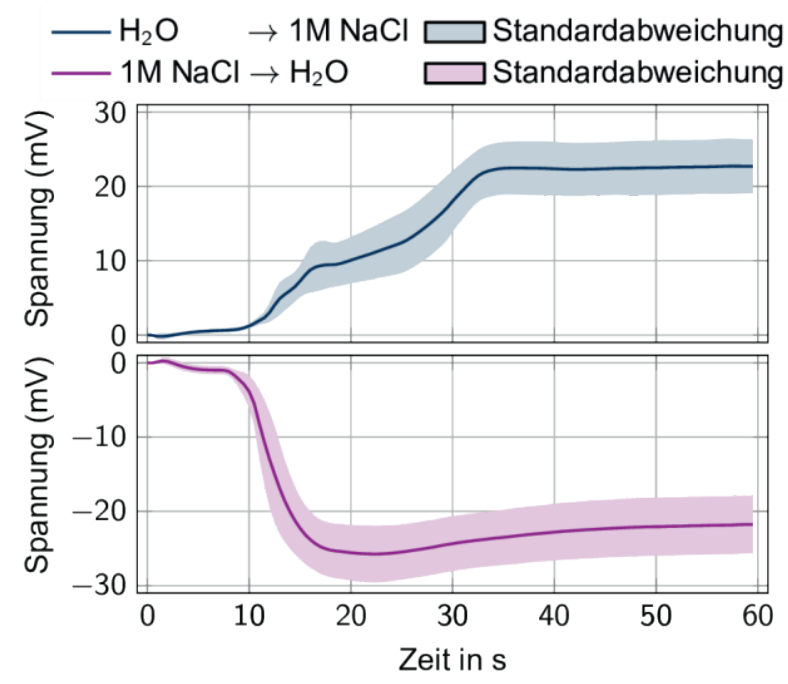

Abb. 7: Mittelwerte und Standardabweichungen der Sprungantworten bei Konzentrationsänderung gemäß Abbildung 6 


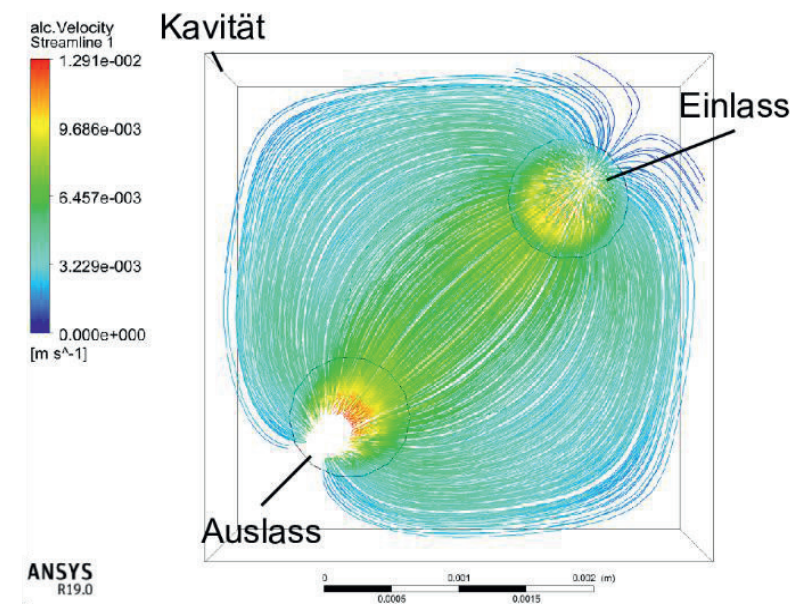

Abb. 8: Simulation des Strömungsverhaltens des Fluids in der Kavität des Sensors (Draufsicht)

Analyts. Zudem ist der Sensoraufbau, wie in Abbildung 3 gezeigt, so gestaltet, dass das Analyt die Kaltseite der Peltierelemente passiert, bevor das Hydrogel erreicht wird, sodass die Temperatur des einströmenden Analyts von der aktuellen Peltierheizleistung abhängig ist.

Außerdem ist noch die Position der Fluidkanäle zu verbessern, um zu verhindern, dass sich hinter den Einlässen in der Kavität Bereiche bilden, welche verhindern, dass das Analyt vollends ausgespült wird und somit nach dem Konzentrationswechsel noch Reste des vorangegangenen Analyts vorhanden sind (Abbildung 8).

\section{Zusammenfassung und Ausblick}

Zielstellung dieses Beitrags war es, einen Hydrogelsensor herzustellen, welcher mit einem sehr schnellen Antwortverhalten die sonst übliche lange Messdauer wesentlich verkürzt.

Dazu wurde der Bimorpheffekt genutzt, wobei das Hydrogel in einem Drucksensor auf einer Siliziumplatte strukturiert ist. Hierfür war es nötig, die Hydrogelzusammensetzung anzupassen, eine Haftung zwischen Hydrogel und Silizium herzustellen und ein geeignetes Verfahren zur Strukturierung des Hydrogels zu finden.

Die Ergebnisse zeigen, dass mit dem neuen Aufbau Sensorreaktionszeiten im Bereich von wenigen Sekunden möglich sind. Die Reproduzierbarkeit der Ergebnisse soll in kommenden Arbeiten durch Verbesserung des Strömungsprofils, Identifikation und Eliminierung der Störeinflüsse sowie Optimierung der Hydrogelzusammensetzung, weiter verbessert werden.

\section{Literatur}

[1] A. KATCHALSKY, Rapid Swelling and Deswelling of Reversible Gels of Polymeric Acids by Ionization, Experientia, 1949, S. 319-320

[2] G. GERLACH und K.-F. ARNDT; Hrsg, Hydrogel Sensors and Actuators. Heidelberg ; New York: Springer, 2009.

[3] J. ERFKAMP, M. GUENTHER, und G. GER$\mathrm{LACH}$, Enzyme-Functionalized Piezoresistive Hydrogel Biosensors for the Detection of Urea, Sensors, 2019, S. 2858

[4] J. ERFKAMP, M. GUENTHER, und G. GERLACH, Hydrogel-Based Sensors for Ethanol Detection in Alcoholic Beverages, Sensors, 2019, S. 1199

[5] J. ERFKAMP, M. GUENTHER, und G. GERLACH, Piezoresistive Hydrogel-Based Sensors for the Detection of Ammonia, Sensors, 2019, S. 971

[6] D. FRANKE und G. GERLACH, Swelling Studies of Porous and Nonporous Semi-IPN Hydrogels for Sensor and Actuator Applications, Micromachines, 2020, S. 425

[7] S. BINDER und G. GERLACH, Intramolecular Force-compensated Hydrogel-based Sensors with Reduced Response Times, Technisches Messen, 2019, S. 227-236

[8] N. GULNIZKIJ und G. GERLACH, Modelling and model verification of an autonomous threshold sensor for humidity measurements, J. Sens. Sens. Syst., 2020, S. 1-6

[9] N. GULNIZKIJ und G. GERLACH, Bistable Threshold Humidity Sensor Switch with Rectangular Bimorph Bending Plate, Micromachines, 2020, S. 569

[10] R: The R Project for Statistical Computing. [Online]. Verfügbar unter: https://www.rproject.org/

\section{Danksagung}

Die Autoren danken der Deutschen Forschungsgemeinschaft (DFG) für die finanzielle Unterstützung im Rahmen des Graduiertenkollegs „Hydrogel-basierte Mikrosysteme“ (GRK 1865). 